Millena Luiza Palhano Jéssica Pinto Mauda Gerusa Clazer Halila Possagno Sinvaldo Baglie Edmar Miyoshi

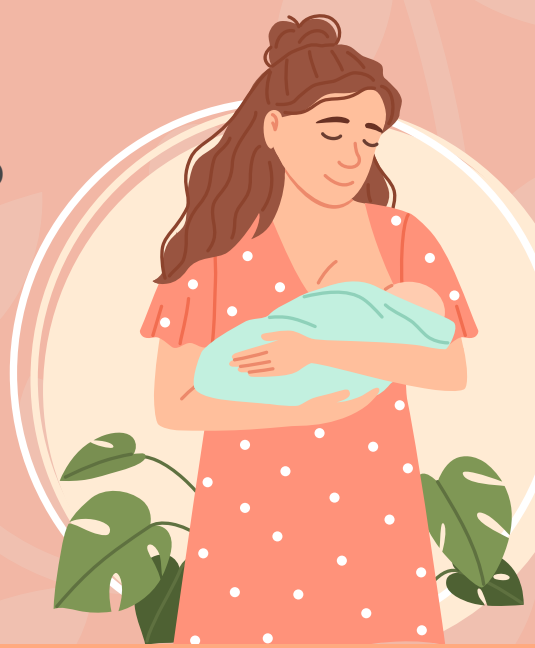

\title{
Orientações farmacêuticas \\ Uso de medicamentos durante o aleitamento materno
}
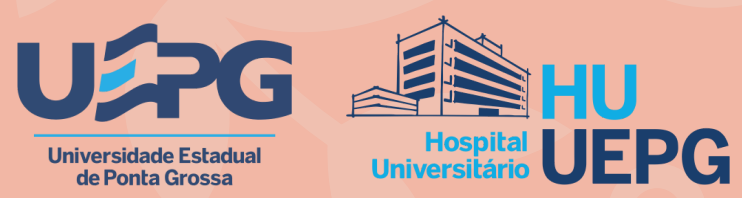


\title{
UNIVERSIDADE ESTADUAL DE PONTA GROSSA
}

\author{
Reitor \\ Miguel Sanches Neto \\ Vice-Reitor \\ Everson Augusto Krum
}

Pró-Reitoria de Extensão e Assuntos Culturais Édina Schimanski 


\section{Millena Luiza Palhano}

\section{Jéssica Pinto Mauda}

\section{Gerusa Clazer Halila Possagno}

Sinvaldo Baglie

Edmar Miyoshi
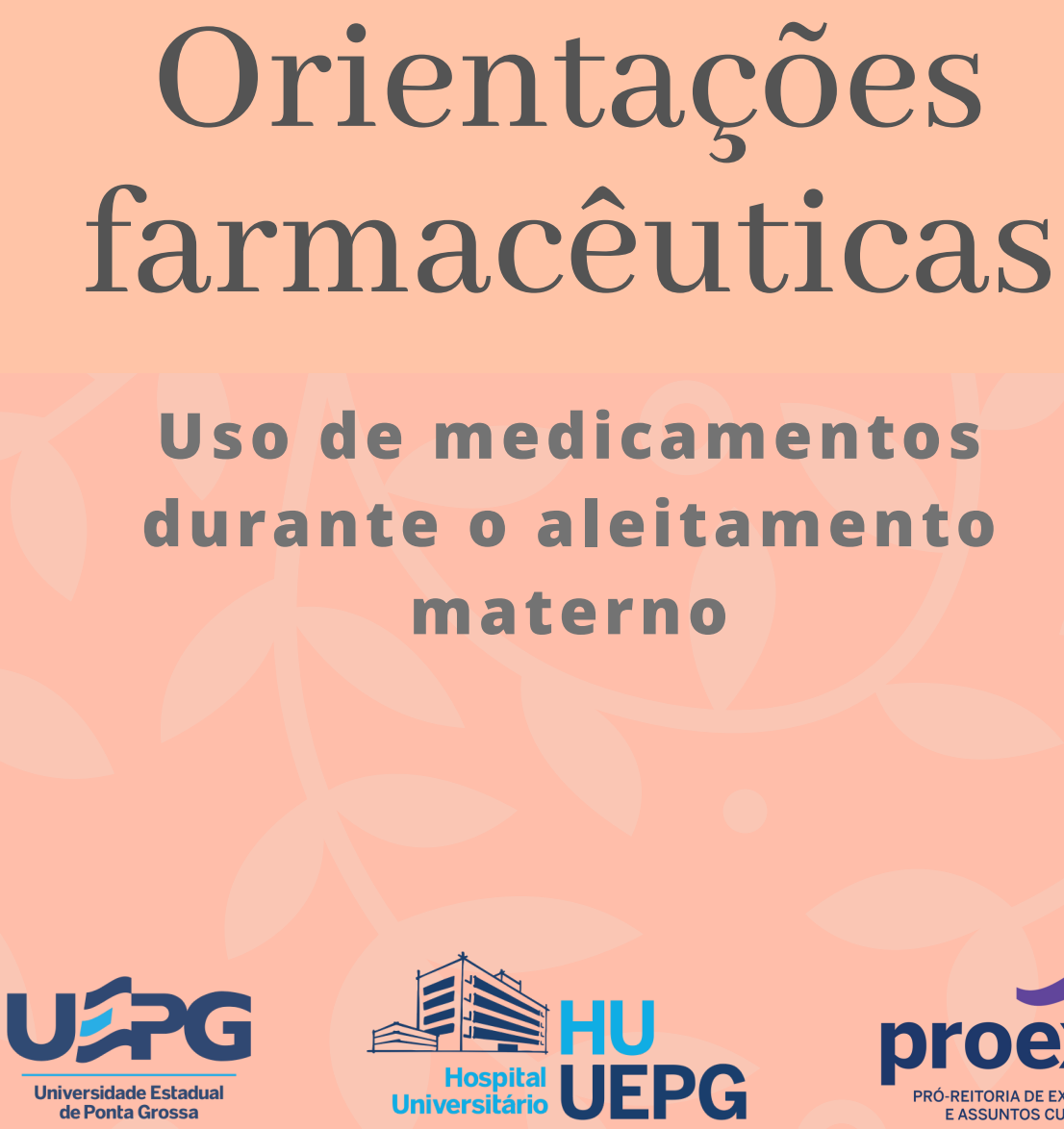


\title{
PROEX UEPG
}

\section{Equipe Editorial}

\section{Revisão de língua portuguesa Emilson Richard Werner}

\author{
Arte \\ Jéssica Pinto Mauda \\ Millena Luiza Palhano
}

\section{Apoio \\ Editora UEPG}

O69

Orientações farmacêuticas: uso de medicamentos durante o aleitamento materno [Livro eletrônico]/ Millena Luiza Palhano et al.

Ponta Grossa: UEPG/PROEX, 2021.

19p.; E-book PDF

ISBN: 978-65-86967-32-6

DOI: $10.212 / 86967-32-6$

1. Amamentação. 2. Medicação. 3. Medicamentos. I. Mauda, Jéssica et al. II. T.

CDD: 617.6

Ficha catalográfica elaborada por Maria Luzia F. B. dos Santos CRB 9/986 


\section{A IMPORTÂNCIA DA AMAMENTAÇĀO}

- A amamentação é a nutrição ideal para o desenvolvimento e crescimento saudável dos bebês;

- Ela é recomendada pela Organização Mundial da Saúde como alimento exclusivo nos primeiros 6 meses;

- Ela favorece a relação afetiva entre mãe e filho;

- Ela melhora o desenvolvimento cognitivo e psicomotor da criança;

- Ela protege contra infecções, alergias, doenças respiratórias, além de reduzir as chances de desenvolver doenças crônicas;

- Ela reduz a mortalidade neonatal;

- Ela não provoca gasto, reduzindo o impacto na renda familiar.

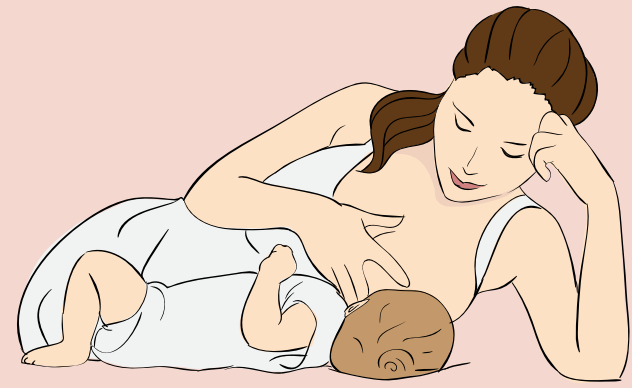




\section{MITO OU VERDADE?}

\section{A maior parte dos medicamentos sāo incompatíveis com 0 aleitamento materno?}

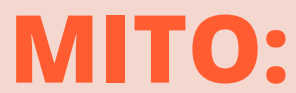

A maioria dos medicamentos são considerados compatíveis

e podem ser utilizados durante a amamentação.

\section{Preciso realizar o desmame do bebê se estou utilizando algum medicamento?}

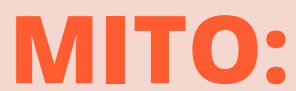

Se você utiliza algum medicamento, não é necessário realizar o desmame do seu bebê se não houver a orientação de um profissional da saúde para isso.

\section{Preciso ter cuidado na utilizaçāo de plantas medicinais durante a amamentaçāo?}

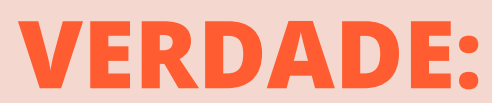

Deve-se ter muito cuidado na utilização de chás ou medicamentos de plantas medicinais, alguns ainda não estudados em relação aos riscos para o bebê. Evite a utilização sem orientação de profissional da saúde. 


\section{ÁLCOOL, NICOTINAE OUTRAS DROGAS}

(1) O álcool e a nicotina presente no cigarro apresentam risco de redução na produção do leite materno;

Ambos podem modificar o odor e o sabor do leite materno, levando à recusa do leite pelo bebê;

(1) Os medicamentos (adesivo, goma de mascar e spray) que contêm nicotina são compatíveis com a amamentação e podem ser utilizados para substituir o cigarro;

(1) O álcool é perigoso para o bebê durante o período da lactação, já a nicotina pode ser compatível com a amamentação, porém deve-se ter cuidado no uso;

(1) Drogas ilícitas como cocaína, crack e maconha são consideradas altamente perigosas e incompatíveis com a amamentação.

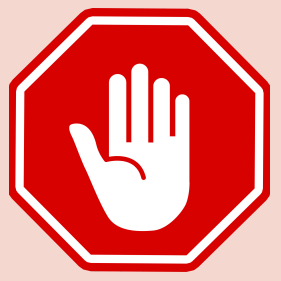

\section{Nāo interrompa 0 aleitamento materno sem a} orientaçāo de um profissional. 


\section{FITOTERÁPICOS \\ E AMAMENTAÇĀO}

Deve-se ter cuidado no uso

abusivo de chás e medicamentos à base de plantas.
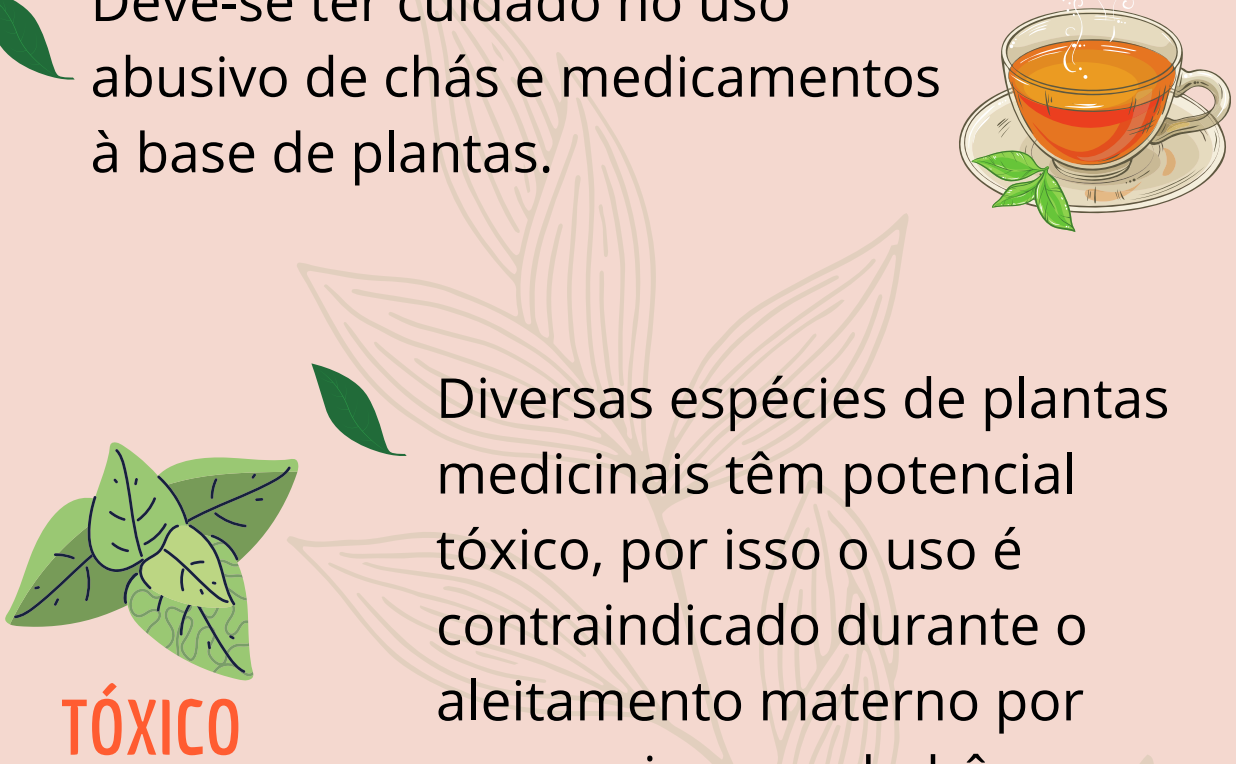

Diversas espécies de plantas medicinais têm potencial tóxico, por isso o uso é contraindicado durante o aleitamento materno por causar riscos ao bebê.

As plantas medicinais devem apresentar estudos de comprovação de eficácia e segurança durante a amamentação para serem utilizadas.

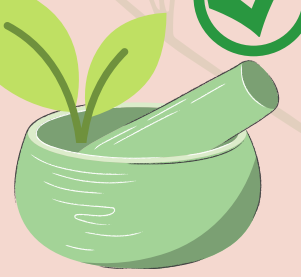




\section{PLANTAS CONTRAINDICADAS:}

\section{Algumas plantas bastante conhecidas sāo}

\section{contraindicadas durante a 0 aleitamento materno, como:}

$\rightarrow$ Alho: pode provocar cólicas no bebê.

$\rightarrow$ Sene: pode causar desconforto gastrointestinal e diarreia na mãe e no bebê.

$\rightarrow$ Alcachofra: pode causar sabor amargo no leite.

$\rightarrow$ Cavalinha: pode causar deficiência de vitaminas.

$\rightarrow$ Lavanda: pode ter efeito tóxico quando ingerida.

$\rightarrow$ Sálvia: pode causar redução do leite.

$\rightarrow$ Boldo-do-chile: pode causar neurotoxicidade.

$\rightarrow$ Erva-mate: pode causar irritabilidade, náuseas e vômitos.

$\rightarrow$ Quebra-pedra: pode causar cólicas e diarreia no bebê.

$\rightarrow$ Cáscara sagrada: pode causar cólicas e diarreia no bebê.

$\rightarrow$ Hortelã: pode inibir a absorção de ferro e causar anemia. Risco aumentado de reação alérgica no bebê.

$\rightarrow$ Tanaceto ou catinga-de-mulata: pode causar alteração do sabor do leite. 


\section{MEDICAMENTOS PERMITIDOS DURANTE A AMAMENTAÇĀO}

O uso de medicamentos durante a amamentação não deve ser considerado um fator de risco.

1- Muitos medicamentos são considerados seguros e permitidos neste período.

$1-$ Por isso, as mães que fazem uso contínuo de medicamentos podem continuar com o tratamento, desde que os medicamentos sejam seguros durante a amamentação.

Nāo interrompa a amamentaçāo e o tratamento de forma precoce sem orientaçāo de um profissional de saúde. 


\section{CUIDADOS COM O USO DE MEDICAMENTOS DURANTE 0 ALEITAMENTO MATERNO}

Observar o bebê sobre possíveis efeitos colaterais dos medicamentos utilizados, como:

alteração da alimentação; mudança de hábitos de sono; agitação;

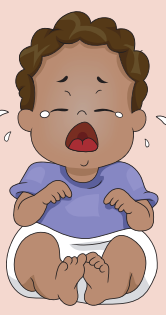

distúrbios gastrointestinais como diarreia ou constipação;

alteração de tônus muscular: rigidez ou relaxamento muscular alterado.

Programar o horário de administração do medicamento imediatamente após a amamentação.

Utilizar medicamentos prescritos por profissionais da saúde e não praticar a automedicação. 


\section{MEDICAMENTOS PERMITIDOS DURANTE A AMAMENTAÇĀO}

\section{Analgésicos:}

Paracetamol e Ibuprofeno.

Ácido Mefenâmico, Cetoprofeno e

Diclofenaco também são

considerados seguros.
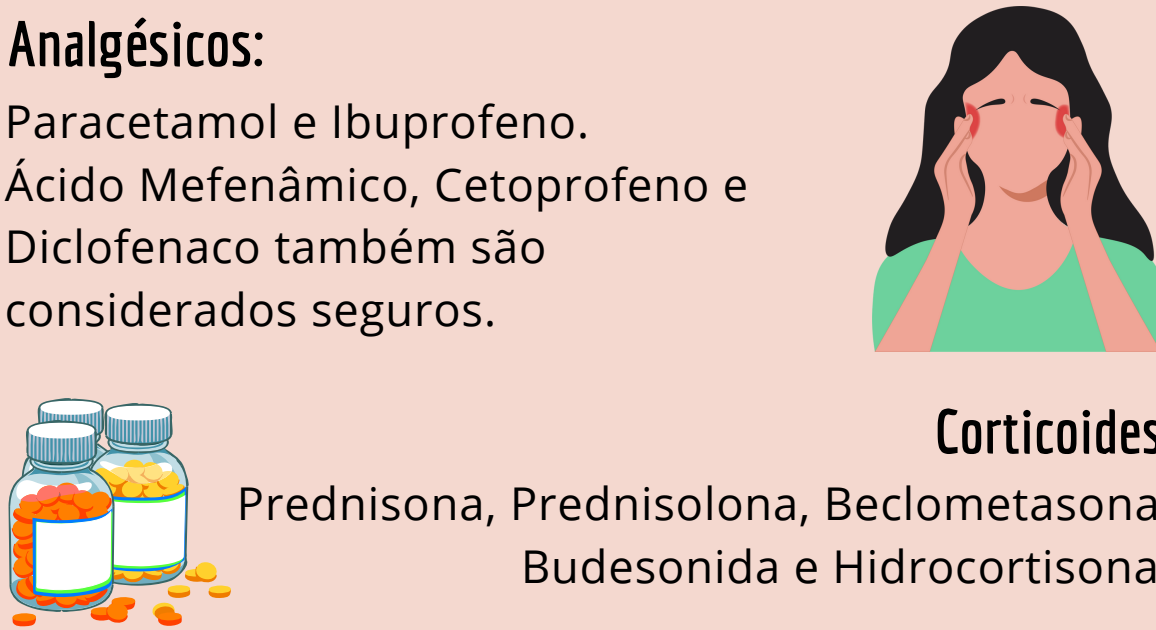

Corticoides:

Prednisona, Prednisolona, Beclometasona, Budesonida e Hidrocortisona.

\section{Antieméticos e gastrocinéticos:}

Domperidona, Metoclopramida e Ondansetrona.

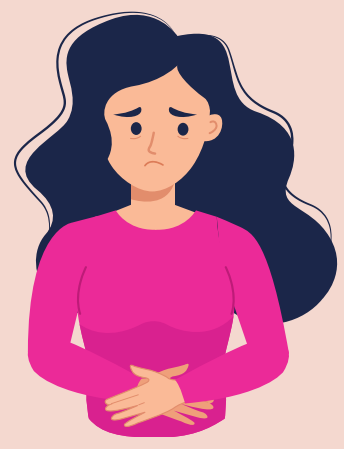

\section{Vitaminas e Minerais:}

Ácido Fólico (Vitamina B9) e demais vitaminas do complexo B, Vitamina C, Vitamina $\mathrm{D}$, Vitamina $\mathrm{E}$, Vitamina $\mathrm{K}$ e Zinco. 


\section{MEDICAMENTOS PERMITIDOS DURANTE A AMAMENTAÇĀO}

Anti-histamínicos:

Orientações: Alguns medicamentos

podem suprimir a lactação.

$1^{\text {a }}$ escolha: Loratadina,

Desloratadina, Fexofenadina, Hidroxizina.

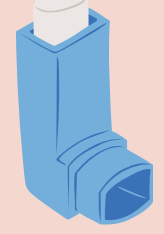

Antiasmáticos:
Brometo de Ipratrópio, Budesonida,
Salbutamol, Propionato de Fluticasona.

\section{Antiácidos:}

Bicarbonato de Sódio, Carbonato de Cálcio, Hidróxido de Magnésio, Hidróxido de Alumínio, Omeprazol.
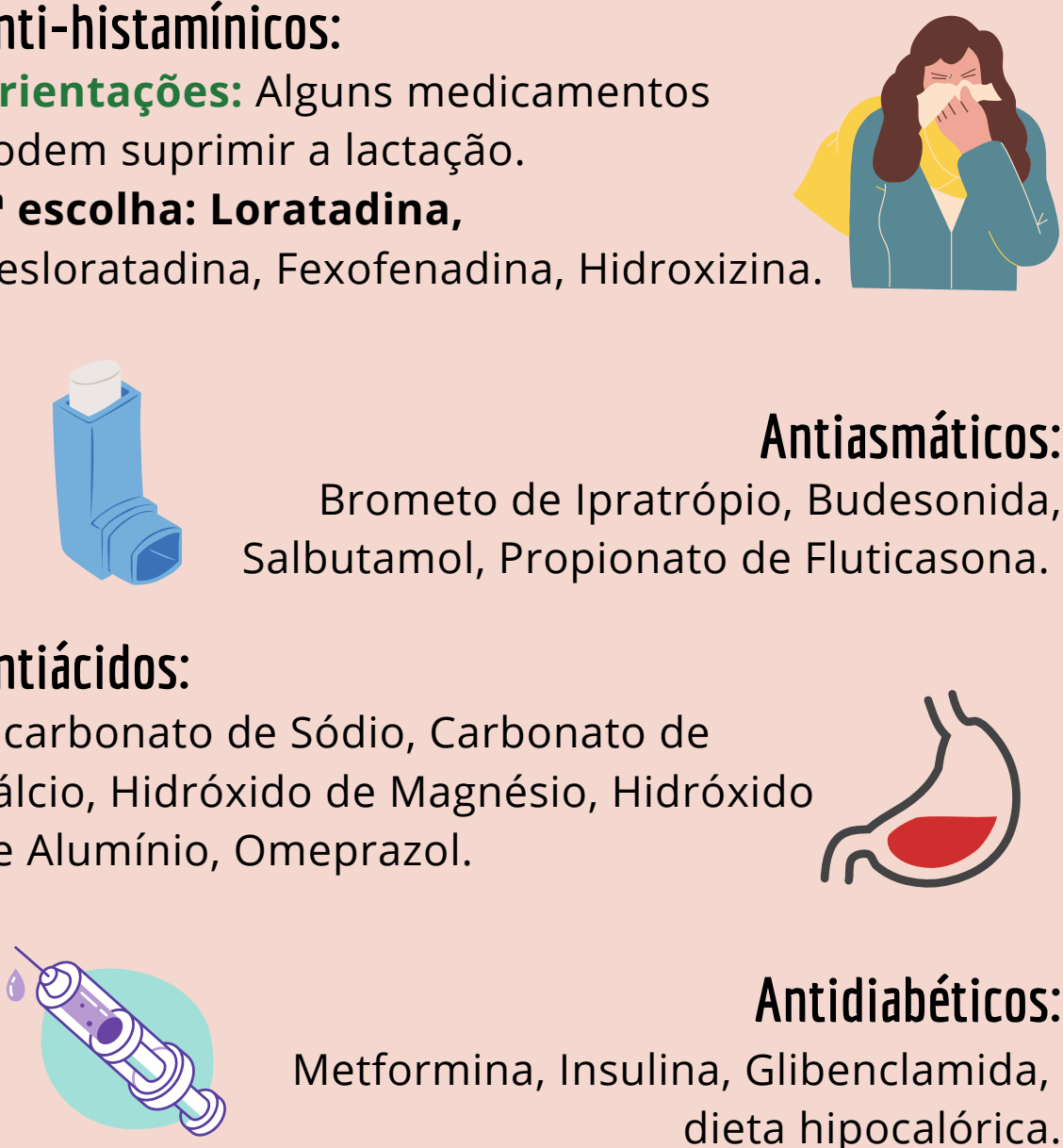

\section{Antidiabéticos:}

Metformina, Insulina, Glibenclamida, dieta hipocalórica.

\section{Antianêmicos:}

Ácido folínico, Ácido fólico, Sulfato ferroso. 


\section{MEDICAMENTOS PERMITIDOS DURANTE A AMAMENTAÇĀO}

Hormônios Tireoideanos e Fármacos Antitireoideanos:

Levotiroxina, Propiltiouracil, Metimazol e Tirotropina.

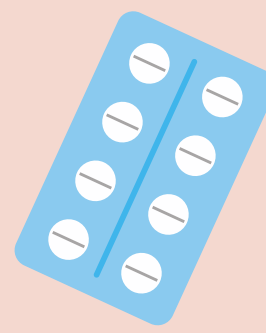

\section{Antimicrobianos/antibióticos:}

Não interromper o tratamento prescrito. Amoxicilina, Amoxicilina + Clavulanato de Potássio, Azitromicina, Cefalexina, Clindamicina, Nitrofurantoína.

\section{Anticonvulsivantes:}

Nunca interromper o tratamento sem orientação médica. Diversos deles são de uso seguro durante a amamentação, como:

Carbamazepina, Ácido Valpróico, Diazepam, Fenitoína e Gabapentina.

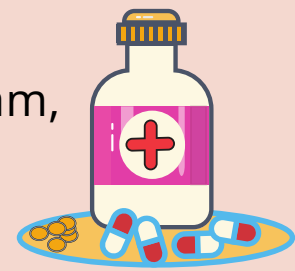




\section{MEDICAMENTOS PERMITIDOS DURANTE A AMAMENTAÇĀO}

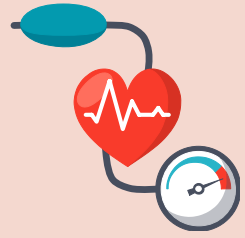

\section{Anti-hipertensivos:}

Captopril, Enalapril, Metildopa, Metoprolol e Propranolol.

\section{Contraceptivos:}

Deve-se optar por aqueles que contêm somente um componente.

Iniciar ou retornar o uso na sexta semana após o parto. Permitidos preferenciais: Desogestrel, Acetato de Medroxiprogesterona.

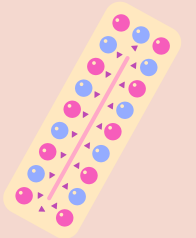

Métodos de barreira, como camisinha masculina ou feminina, diafragma e DIU podem ser utilizados.

\section{Antidepressivos:}

Não devem ser interrompidos de forma abrupta. Muitos deles são seguros na amamentação, como:

Amitriptilina, Citalopram, Escitalopram, Nortriptilina, Paroxetina e Sertralina. 


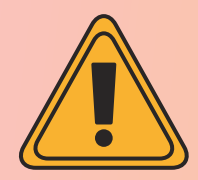

Relaxantes musculares

\section{Diuréticos}

\section{Descongestionantes} nasais

Analgésicos

\section{CUIDADOS}

O Dorflex $\circledR^{\circledR}$, comumente utilizado, tem em sua composição o Citrato de Orfenadrina, e o Torsilax ${ }^{\circledR}$ tem

Carisoprodol, que não são recomendados para uso durante a amamentação.

Diuréticos como Amilorida, Clortalidona, Furosemida e Indapamida devem ser evitados na lactação, pois podem diminuir a produção do leite materno.

Benegrip Multi ${ }^{\circ}$, Decongex Plus ${ }^{\circ}$, Naldecon $®$ e outros apresentam em sua composição Fenilefrina, a qual é contraindicada durante a amamentação, pois pode diminuir a produção do leite materno.

Neosaldina $₫$, Doralgina $₫$, Sedamed $\AA$ e outros apresentam em sua composição Mucato de Isometepteno, o qual pede cuidado criterioso para uso durante a amamentação. 


\section{ORIENTAÇŌES}

Medicamentos tópicos, como cremes e pomadas, nāo sāo excretados no leite materno em quantidades significativas, porém é necessário ter cautela se forem usados na regiāo da mama.

Alguns medicamentos apresentam vários componentes, leia sempre atentamente a bula para identificar se todos os ingredientes ativos presentes nos medicamentos sāo seguros antes de usá-los.

Alguns medicamentos considerados seguros podem alterar 0 sabor do leite materno. Recomenda-se observar a aceitaçāo do bebê.
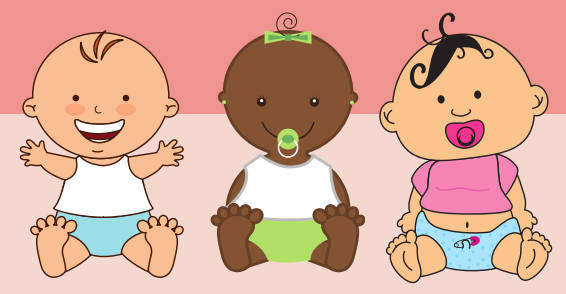


\section{REFERÊNCIAS}

BRASIL. Ministério da Saúde. Ministério da Saúde lança campanha de incentivo à amamentação. Disponível em: https://www.gov.br/pt-br/noticias/saude-e-vigilanciasanitaria/2020/08/ministerio-da-saude-lanca-campanha-deincentivo-a-amamentacao. Acesso em: 18 de jun. de 2021.

BRASIL. Ministério da Saúde. Secretaria da Atenção à Saúde.

\section{Amamentação e uso de medicamentos e outras}

substâncias. 2. ed. Brasília: Ministério da Saúde, 2010.

CASALI, J. M.; PEREIRA, R. J. Uso de plantas medicinais na lactação: uma discussão sobre segurança. Enciclopédia biosfera. Goiânia, v. 16 n. 29; p. 21-56, 2019. Disponível em: https://conhecer.org.br/ojs/index.php/biosfera/article/view/33 4. Acesso em: 20 jul. 2021.

DEPARTAMENTO CIENTÍFICO DE ALEITAMENTO MATERNO. Amamentação: A base da vida. Sociedade Brasileira de Pediatria, Rio de Janeiro, v. 6, p. 1-4, 2018. Disponível em: https://www.sbp.com.br/imprensa/detalhe/nid/amamentacao -a-base-da-vida/. Acesso em: 20 jul. 2021.

DEPARTAMENTO CIENTÍFICO DE ALEITAMENTO MATERNO. Uso de medicamentos e outras substâncias pela mulher durante a amamentação. Sociedade Brasileira de Pediatria. v. 4, p. 1-18, ago. 2017. Disponível em: https://www.sbp.com.br/fileadmin/user_upload/Aleitamento__Uso_Medicam_durante_Amament.pdf. Acesso em: 19 jul. 2021.

IS THIS compatible with breastfeeding? E-lactancia. 9 jul. 2021. Disponível em: http://www.e-lactancia.org/. Acesso em: 18 jun. 2021. 
RAMINELLI, M.; HAHN, S. R. Medicamentos na amamentação: quais as evidências? Ciência e Saúde Coletiva, Rio de Janeiro, n. 24, v. 2, p. 573-587, fev. 2019. Disponível em: https://doi.org/10.1590/1413-81232018242.30052016. Acesso em: 19 jul. 2021.

\section{SECRETARIA DO ESTADO DE SAÚDE. Resolução SES/RJ n.}

1757, de 18 de fevereiro de 2002. Contra-indica o uso de plantas medicinais no âmbito do estado do Rio de Janeiro e dá outras providências. Rio de Janeiro: Secretaria de Estado da Saúde, 18 fev. 2002. Disponível em:

https://antigo.saude.gov.br/images/pdf/2019/janeiro/14/resol ucao-ses-rj-1757-18-2-2002-contra-indica-plantasmedicinais.pdf. Acesso em: 18 jun. 2021.

SILVA, J. B. da; BATISTA, A. M. Avaliação do uso de medicamentos por puérperas no pós-parto normal e cesárea em relação aos riscos sobre a lactação em um hospital do Rio Grande do Norte, Brasil. Infarma: Ciências Farmacêuticas, Brasília, DF, v. 31, n. 2, p. 104-112, 2019. Disponível em: http://www.revistas.cff.org.br/? journal=infarma\&page=article\&op=view $\&$ path $\% 5 B \% 5 D=2501$. Acesso em: 20 jul. 2021. 